\title{
Development Identification Method of Inertia Properties for Heavy Truck Engine Based on MIMS Test Rig
}

\author{
Tianjun Zhu ${ }^{1,3}$, Fudong Zhang ${ }^{1}$, Jianying $\mathrm{Li}^{1}$, Fei Li ${ }^{2}$, Changfu Zong ${ }^{1}$ \\ ${ }^{1}$ Department of Mechanical and Automotive Engineering, Zhaoqing Univeristy, Zhaoqing, China \\ ${ }^{2}$ China Automotive Technology and Research Center, Tianjin 300300, China \\ ${ }^{3}$ Department of Mechanical and Equipment Engineering, Hebei University of Engineering, Handan, 056021, China
}

\begin{abstract}
A new development for the accurate measurement of the inertia parameters for heavy truck engine is presented. It is specifically intended for measuring the inertia properties of vehicle bodies, and it has the potential to be applied to the measurement of the properties of vehicle bodies, such as the vehicle powertrain, engine, and gearbox. This paper, based on CATARC MIMS test rig, develops an accurate measuring method to identify inertia parameters of heavy truck engine. Firstly corresponding tests are carried out and the lever principle and moments of inertia parallel theorem are employed to calculate and analyze the test results, which leads to the accurate value of inertia parameters. Secondly the accuracy of proposed method is verified through the calibration system. As a result the method shows high accuracy, which provides an experimental basis for accurate inertia parameters measurement of heavy truck engine.
\end{abstract}

\section{Introduction}

Modern automobile technology used to isolate the vibration caused by vehicle engine is usually elastic suspension system, which effectively reduces the vehicle vibration and noises leading to the improvements in ride comfort and vehicle quality. Consequently, the design and optimization analysis of vehicle engine mounting and suspension system has been a tough issue and received constant concern in the modern vehicle development and research area. While the design of heavy truck engine mounting and suspension system includes more than mounting position, static and dynamic stiffness and damping parameters design, it is also closely related to the inertia parameters of heavy truck engine such as the position of the center gravity, moment of inertia. The application of the inertia parameters test rig enables the accurate measurement of inertia parameters, then it provides the basic parameters required in the establishment of heavy truck model and the design of heavy truck engine mounting and suspension system, so as to make a valid way for the heavy truck engine dynamics analysis and suspension system optimization design.

In recent studies, researchers and automotive manufactures pay close attention to the inertia parameters measurement technique, which plays an important role in the dynamic performance of vehicle comfort and drivability[1]-[3]. The methodologies for measuring the C.G position and inertia tensor of vehicle bodies are usually divided into three categories: calculation method based on 3D solid model [4], multi-cable pendulum[5], trifilar torsional pendulum[6]-[7]. It is a huge and difficult job to accomplish the 3D solid modelling of vehicle bodies, such as powertrain, engine and gearbox. Hence the calculation method based on the CAD model is not popular used. The most reliable way to get accurate estimation of the inertia properties is via experimental tests. Among the inertia properties only the mass is indirectly measurement with very high accuracy[8]-[9]. The center of gravity can be identified by complex test rigs. For exmaple, M. Gobbi[5] uses the non-linear motion of multi-cable pendulum to measure the inertia properties of rigid bodies. A validation of the measurement procedure is performed by means of experimental tests. Raquel A.B. Almeida[10] provides the group of modal methods, where the rigid body properties are estimated from a modal analysis identification procedure. Renato Brancati[11] proposes an equipment and a procedure for the determination of large object inertia parameters. Measurement is based on the application of a least-square optimization technique on data from force and displacement sensors on the test bench. The ABD company [12] develops the Moment of Inertia (MOI) test system. The MOI test system is capable of finding the position of the center of gravity of the body, the mass and principal inertias, and the orientation of the principal axes. In the latter case, better accuracy would be expected, however the identification of the center of gravity location is a direct operation only for the two coordinates in the horizontal plane. For the third coordinate, if the rigid bodies cannot 
be rotated at will, quite complicated approaches are needed and poor measurement accuracy is to be expected [13].

The paper is organized as follows. At first the hardware of the test rig is introduced, and then the lever principle and moments of inertia parallel theorem are employed to calculate and analyze the test results. A validation of the measurement procedure is performed by means of experimental tests. Finally, Error analysis and the technical specifications of the test rig are given.

\section{Test hardware system}

\subsection{The MIMS test system}

The Moment of Inertia Measurement System (MIMS) is an enhancement to ABD's Suspension Parameter Measurement Machine (SPMM), which extends the machine's capability beyond its original Kinematics and Compliance testing role [13]. As illustrated in Figure 1, MIMS inertia parameters test system based on K\&C test rig in CARTAR, with four six axis force calipers (see Figure 2), and can attach the vehicle bodies to the central table of the test rig where carries out six axis motions (X $、 \mathrm{Y} 、 \mathrm{Z}$, roll, pitch and yaw).A series of test conditions are exerted by six electrical cylinders and the relevant parameters can be figured out through calculating the data generated in the calipers. The excitation is pseudo-static for measurement of the center of gravity height, and is applied at $1 \mathrm{~Hz}$ (typically) for inertia measurements.

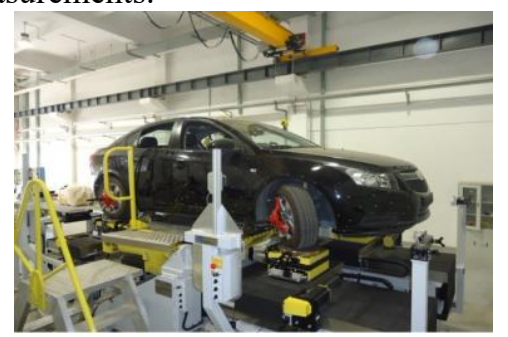

Figure 1. The moment of inertia measurement system test rig

\subsection{The inertia parameters test system of truck engine}

As the heavy truck engine is composed of irregular parts, it is difficult to calculate the inertia parameters, which makes it obtainable through the inertia parameters testing system. The proposed test system takes the heavy truck engine of specific vehicle as example, based on the inertia parameters MIMS test rig, through adjusting the joint angle and length of the test base and heavy truck engine (see Figure 2), and the heavy truck engine is mounted to the test base as in a real vehicle. As illustrated in Figure 3, the first step is to measure the moment of inertia parameters of heavy truck engine and the base; second step is to remove the heavy truck engine, however, as the connecting device is not part of heavy truck engine, it is left together with the base, and to measure the inertia parameters of only the base as illustrated in Figure 4. The test data collected above are put to analysis, then the center of gravity position is identified and the moment of inertia of heavy truck engine can be figured out.

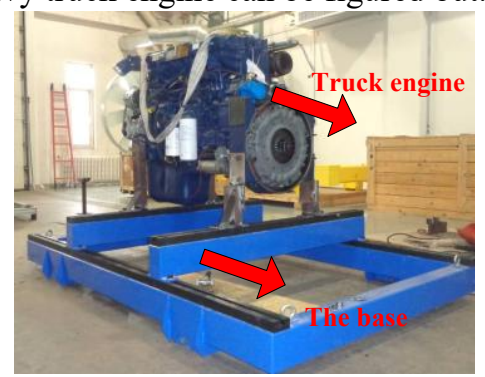

Figure 2. Heavy truck engine installation on the base

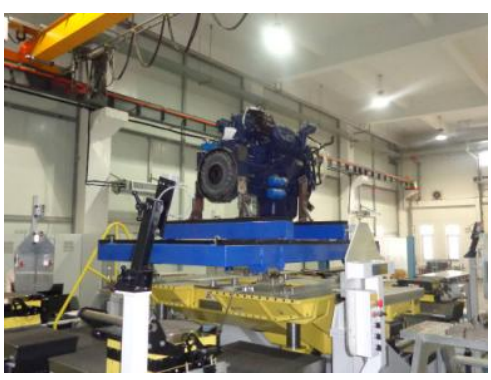

Figure 3. The inertia parameters test system (heavy truck engine + the base)

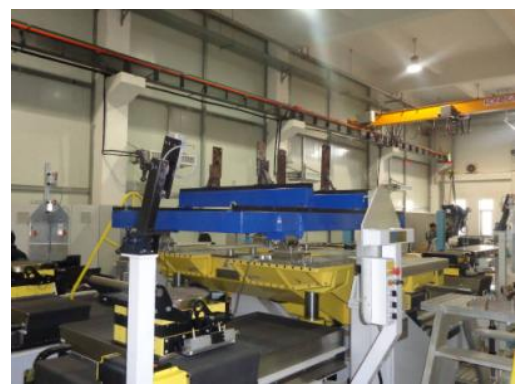

Figure 4. The inertia parameters test system (only the base)

\section{Identification of the inertia properties}

Coordinate system is established according to the right hand rule, choosing the forward direction along the platform as the positive direction of $\mathrm{X}$ axis, the direction to the left as the positive direction of $\mathrm{Y}$ axis, the vertical upward direction as the positive direction as $\mathrm{Z}$ axis, and the origin coordinate point as the center symmetric point. The identification procedure of MOI parameters is as the following:

(1) Step 1: According to the inertia parameters test data (heavy truck engine and the base together) and measured data of only the base, the center of gravity position of heavy truck engine can be identified. The calculating lever principle is shown as the follow.

$$
\begin{aligned}
& m X_{C G}=m_{1} X_{C G 1}+m_{2} X_{C G 2} \\
& m Y_{C G}=m_{1} Y_{C G 1}+m_{2} Y_{C G 2} \\
& m Z_{C G}=m_{1} Z_{C G 1}+m_{2} Z_{C G 2}
\end{aligned}
$$

Where, $m$ is the mass of body (heavy truck engine and the base together); $m_{1}$ is the mass of body (heavy truck engine) and $m_{2}$ is the mass of body (the base) respectively; 
$\left(X_{C G}, Y_{C G}, Z_{C G}\right)$ are the distances from the C.G point of the body (heavy truck engine and the base together) to the origin coordinate of the test rig along three directions; $\left(X_{C G 1}, Y_{C G 1}, Z_{C G 1}\right)$ are the distances from the C.G point of body (heavy truck engine) to the origin coordinate of test rig along three directions; $\left(X_{C G 2}, Y_{C G 2}, Z_{C G 2}\right)$ are the distances from the C.G point of body (the base) to the origin coordinate of test rig along three directions. From the above equations, the C.G point position $\left(X_{C G}, Y_{C G}, Z_{C G}\right)$ relative to the origin coordinate of the test rig can be identified.

(2)Step 2: According to the parallel axis theorem, the body of mass is made to rotate about an axis passing through the body's center of mass. The body (heavy truck engine and the base together) has a moment of inertia $J_{0}$ with respect to this axis. The parallel axis theorem states that if the body is made to rotate instead about a new axis which is parallel to the first axis and displaced from it by a distance $d$, then the moment of inertia $J$ with the new axis is related to $J_{0}$ by

$$
J=J_{0}+m d^{2}
$$

The product of inertia $J_{x y} 、 J_{x z} 、 J_{y z}$ have the similar calculation as the follow

$$
\begin{aligned}
& J_{x y}=J_{x y 0}+m x_{1} y_{1} \\
& J_{x z}=J_{x z 0}+m x_{1} z_{1} \\
& J_{y z}=J_{y z 0}+m y_{1} z_{1}
\end{aligned}
$$

Where, $J_{x y} 、 J_{x z} 、 J_{y z}$ are the products of inertia of the body, and $J_{x y 0} 、 J_{x z 0}, J_{y z 0}$ are the products of inertia of the body which origin coordinate point moves to reference point $\mathrm{R}\left(x_{1}, y_{1}, z_{l}\right)$.

(3) Step 3: Working on the same principle, the parallel axis theorem is used to figure out the moment of inertia of only the base body (products of inertia is included) in the test rig coordinate system.

(4) Step 4: The moment of inertia (products of inertia is included) obtained from step 2 is used to minus the moment of inertia (products of inertia is included) obtained from step 3, and the result is the moment of inertia of heavy truck engine (products of inertia is included) in the test rig coordinate system.

(5) The CG/MOI testing process takes less than 10 minutes.

\section{Test results}

\subsection{The test results of mass and C.G position}

Based on the Inertia parameters measuring test rig, the experimental case study is illustrated in Figure 5, with the corresponding location of the reference point. In the test rig measuring system, the multi-axis load cells used are piezoelectric devices manufactured by Kistler. Piezoelectric are used because of their unique ability to measure small forces accurately in the presence of the large static load generated by the weight of the vehicle bodies, such as heavy truck engine. The test excitation is pseudo-static for measurement of the center of gravity height, and is applied at $1 \mathrm{~Hz}$ (typically) for inertia parameters measurements. The measurement result of mass and C.G. position is shown in Table 1.

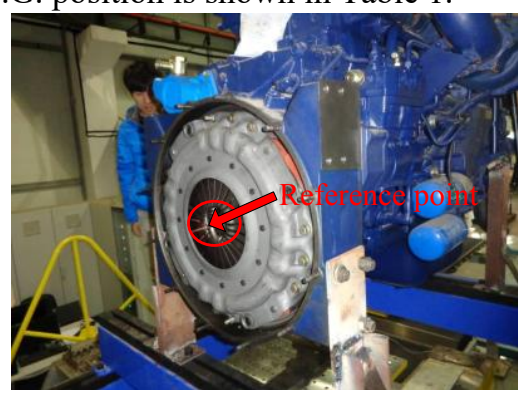

Figure 5. CATARC test rig during the experimental case

Table 1. The experimental result of mass and C.G. position

\begin{tabular}{|c|c|c|}
\hline & $\begin{array}{c}\text { heavy Truck } \\
\text { engine+the base }\end{array}$ & the base \\
\hline Mass & $1674.4(\mathrm{~kg})$ & $459.8(\mathrm{~kg})$ \\
\hline $\begin{array}{c}\text { C.G. position } \\
\left(X_{C G}\right)\end{array}$ & $-76.5(\mathrm{~mm})$ & $-5.6(\mathrm{~mm})$ \\
\hline $\begin{array}{c}\text { C.G. position } \\
\left(Y_{C G}\right)\end{array}$ & $5.4(\mathrm{~mm})$ & $-2.7(\mathrm{~mm})$ \\
\hline $\begin{array}{c}\text { C.G. position } \\
\left(Z_{C G}\right)\end{array}$ & $1060.6(\mathrm{~mm})$ & $489.7(\mathrm{~mm})$ \\
\hline
\end{tabular}

From Table 1, it is illustrated that the mass of heavy truck engine and the base is $1674.4 \mathrm{~kg}$, and the center of gravity is $76.5 \mathrm{~mm}$ backward from the test rig origin coordinate point, $5.4 \mathrm{~mm}$ to the left and $1060.6 \mathrm{~mm}$ upward. Simultaneously the mass of only the base body is $459.8 \mathrm{~kg}$ and the center of gravity is $5.6 \mathrm{~mm}$ backward from the test rig origin coordinate point, $2.7 \mathrm{~mm}$ to the right and $489.7 \mathrm{~mm}$ upward.

Based on the lever principle according the test data of mass and C.G position, the mass and center of gravity of heavy truck engine are listed in Table 2 choosing the reference point on the heavy truck engine to identify the C.G. position of heavy truck engine.

Table 2. The experimental result of mass and C.G. position (Heavy Truck Engine)

\begin{tabular}{|c|c|c|}
\hline & $\begin{array}{c}\text { heavy Truck } \\
\text { engine+the base }\end{array}$ & $\begin{array}{c}\text { the reference } \\
\text { point }\end{array}$ \\
\hline Mass & $1214.6(\mathrm{~kg})$ & \\
\hline $\begin{array}{c}\text { C.G. position } \\
\left(X_{C G}\right)\end{array}$ & $-103.34(\mathrm{~mm})$ & $-650.34(\mathrm{~mm})$ \\
\hline $\begin{array}{c}\text { C.G. position } \\
\left(Y_{C G}\right)\end{array}$ & $8.47(\mathrm{~mm})$ & $15.47(\mathrm{~mm})$ \\
\hline $\begin{array}{c}\text { C.G. position } \\
\left(Z_{C G}\right)\end{array}$ & $1276.72(\mathrm{~mm})$ & $165.72(\mathrm{~mm})$ \\
\hline
\end{tabular}

From Table 2, the mass of heavy truck engine and the base minus the mass of the base equals the mass of heavy truck engine which is $1214.6 \mathrm{~kg}$, and the center of gravity is $103.34 \mathrm{~mm}$ backward from the test rig origin 
coordinate point, $8.47 \mathrm{~mm}$ to the left and $1276.72 \mathrm{~mm}$ upward.

Notes: The center of the intersection between the center line of engine crankshaft and flywheel housing end plane is chosen as reference point as illustrated in Figure 6 which is marked by a red circle. The position of the reference point in the coordinate system of test rig is $(547,-7,1111)$. As the reference point is the origin coordinate point in the $3 \mathrm{D}$ model coordinate system, so the center of gravity of heavy truck engine in the 3D model system is $(-650.34,15.47,165.72)$.

\subsection{The identification results of inertia parameters}

The experimental data by the Inertia parameters measuring test rig are used in the identification of inertia parameters for heavy truck engine based on the parallel axis theorem. The identification results are shown in Table 3.

Table 3. The identification results of inertia parameters

\begin{tabular}{|c|r|r|r|}
\hline & $\begin{array}{c}\text { heavy Truck } \\
\text { engine+the } \\
\text { base body }\end{array}$ & \multicolumn{1}{|c|}{$\begin{array}{c}\text { heavy } \\
\text { truck } \\
\text { engine }\end{array}$} & \multicolumn{1}{c|}{$\begin{array}{c}\text { the base } \\
\text { body }\end{array}$} \\
\hline$J_{x}\left(\mathrm{kgm}^{2}\right)$ & 477.1 & 81.37 & 189.1 \\
\hline$J_{y}\left(\mathrm{kgm}^{2}\right)$ & 730.3 & 244.8 & 275.7 \\
\hline$J_{z}\left(\mathrm{kgm}^{2}\right)$ & 657.6 & 207.3 & 447.1 \\
\hline$J_{x y}\left(\mathrm{kgm}^{2}\right)$ & -0.8 & -2.14 & 1.7 \\
\hline$J_{x z}\left(\mathrm{kgm}^{2}\right)$ & -34.1 & -8.94 & 0.5 \\
\hline$J_{y z}\left(\mathrm{kgm}^{2}\right)$ & 6.4 & 4.57 & -1.1 \\
\hline
\end{tabular}

From Table 3 , it is shown that heavy truck engine moment of inertia is $81.37 \mathrm{kgm}^{2}$ relative to $X$ axis , $244.8 \mathrm{kgm}^{2}$ relative to $Y$ axis, and $207.3 \mathrm{kgm}^{2}$ relative to $Z$ axis. The three products of inertia of $J_{\mathrm{xy}} 、 J_{\mathrm{xz}} 、 J_{\mathrm{yz}}$ are $2.14 \mathrm{kgm}^{2},-8.94 \mathrm{kgm}^{2}$ and $4.57 \mathrm{kgm}^{2}$ respectively.

\section{Conclusions}

A new approach for the accurate measurement of the inertia parameters for heavy truck engine is presented. Compared to the conventional inertia parameters measurement method, this new approach has several advantages:

(1) By using the MIMS measuring instrument, the measurement accuracy is significantly enhanced.

(2) The calibration procedure with standard specimen showed little deviation from conventionally true values, the developed method showed good repeatability.

(3) The MOI measurement system enables the center of gravity coordinates and inertia tensor components to be measured for kinds of vehicle bodies such as powertrain, truck cabs, engine, gearbox, etc.
(4) The CG/MOI testing process takes less than 10 minutes, so the test method has good working efficiency.

\section{Acknowledgments}

The authors would like to acknowledge the financial support of Collaborative Innovation and Platform Environment Construction Project of Guangdong Province (2015A050502053) , Hebei science and technology plan project(17394501D), Science and technology research project of Hebei higher education (ZD2017213) Hebei provincial High Level Talents Foundation of Hebei Province (A2016002025), Characteristic innovation project of Guangdong Province (2016KTSCX154), The authors also would like to acknowledge the financial support of Natural Science Foundation of Guangdong Province (2014A030311045) and project of Education Department of Guangdong Province.

\section{References}

1. Wen-Bin, Shangguan, Y. Zhao, "Dynamic analysis and design calculation methods for powertrain mounting systems," International Journal of Automotive Technology, Vol. 8 , No. 6 , pp. 731 744, 2007.

2. Rozyn, Matthew, Zhang, Nong, "A method for estimation of vehicle inertial parameters," International Journal of Vehicle System Dynamics, Vol. 48, No. 5, pp. 547-565, 2010.

3. Wegener, Daniel, "Vehicle inertia measurement machine (VIMM)," Society of Allied Weight Engineers - 71st International Conference on Mass Properties, pp. 153-162, 2012.

4. J.P. Pegram, W.A. Anemaat, "Preliminary estimation of airplane moments of inertia using CAD solid modeling," SAE paper 2000-01-1700, 2000.

5. M. Gobbi, G. Mastinu, G. Previati, "A method for measuring the inertia properties of rigid bodies, "Mechanical Systems and Signal Processing, Vol. 25 ,No.1, pp. 305 - 318,2011.

6. S. Carsten, L. Michhael, "A survey of current inertia parameter identification methods, "Mechanical Systems and Signal Processing, Vol. 15, No. 1, pp. 189 - 211, 2001.

7. Zhi-Chao. Hou, Yi-ning. Lv, Yao-xin. Lao, Dan. Liu, "A new trifilar pendulum approach to identify all inertia parameters of a rigid body or assembly," Mechanism and Machine Theory,Vol.44, No.3 pp. 1270 - 1280, 2009.

8. R.A.B. Almeida, A.P.V. Urgueira, N.M.M. Maia, "Evaluation of the performance of three different methods used in the identification of rigid body properties,"Shock and Vibration Journal (Special Issue ICED 2007), pp. 467-479, 2008.

9. H. Lee, Y.B. Lee, Y.S. Park, "Response and excitation points selection for accurate rigid body inertia properties identification," Mechanical 
Systems and Signal Processing,Vol.13,No.4 , pp.571-592,1999.

10. Raquel A.B.Almeida ,Antonio P.V.Urgueira, NunoM.M.Maia, "Further developments on the estimation of rigid body properties from experimental data," Mechanical Systems and Signal Processing,No.4,pp.1391-1408, 2010.

11. Renato Brancati,Riccardo Russo, SergioSavino, "Method and equipment for inertia parameter identification," Mechanical Systems and Signal Processing,No.24,pp. 29-40, 2010.

12. Anthony Best, "Addendum 1 to SPMM User Guide (MIMS) v8.12,"Anthony Best Dynamics Company, pp.63-68, 2012.

13. Xiao Shuchen, Qing Yuxun, Han Jiqing, " A test analysis method based on Grubbs in experiments". Journal of Projectiles; Rockets; Missiles and Guidance, Vol.27, No.1, pp.275-277, 2007. 